\title{
On regular and equivelar Leonardo polyhedra
}

\author{
Gábor Gévay \\ Bolyai Institute, University of Szeged, Aradi vértanúk tere 1, H-6720 Szeged, Hungary \\ Jörg M. Wills
}

Math. Institute, University of Siegen, Emmy-Noether-Campus, D-57068 Siegen, Germany

Received 12 July 2011, accepted 4 January 2012, published online 1 June 2012

\begin{abstract}
A Leonardo polyhedron is a 2-manifold without boundary, embedded in Euclidean 3space $\mathbb{E}^{3}$, built up of convex polygons and with the geometric symmetry (or rotation) group of a Platonic solid and of genus $g \geq 2$. The polyhedra are named in honour of Leonardo's famous illustrations in [19] (cf. also [12]). Only six combinatorially regular Leonardo polyhedra are known: Coxeter's four regular skew polyhedra, and the polyhedral realizations of the regular maps by Klein of genus 3 and by Fricke and Klein of genus 5. In this paper we construct infinite series of equivelar (i.e. locally regular) Leonardo polyhedra, which share some properties with the regular ones, namely the same Schläfli symbols and related topological structure. So the weaker condition of local regularity allows a much greater variety of symmetric polyhedra.
\end{abstract}

Keywords: Equivelar polyhedron, Leonardo polyhedron, regular polyhedron, genus, Schläfli symbol, symmetry group.

Math. Subj. Class.: 52B15, 52B70

\section{Introduction}

A polyhedron is a compact 2-manifold without boundary embedded in Euclidean 3-space $\mathbb{E}^{3}$, hence oriented. It is built up of finitely many (planar) convex polygons, any two of which meet, if at all, in a single edge or a single vertex.

If $v, e$ and $f$ denote the number of vertices, edges and faces, respectively, of the polyhedron, then one has the basic Euler-Poincaré equation

$$
v-e+f=2-2 g=\chi,
$$

E-mail addresses: gevay@math.u-szeged.hu (Gábor Gévay), wills@ mathematik.uni-siegen.de (Jörg M. Wills) 
where $g \geq 0$ denotes the genus and $\chi$ the Euler characteristic. In this paper we do not consider the case of tori $(g=1)$, but only the polyhedra with $g \geq 2$. If all faces of a polyhedron are $p$-gons, $p \geq 3$, and all vertices $q$-valent, $q \geq 3$, then the polyhedron is called locally regular or equivelar and is denoted by its Schläfli symbol $\{p, q\}$ (cf. [3, 15]). We note that the extended Schläfli symbol $\{p, q ; g\}$ is also used. A much stronger condition is (global combinatorial) regularity: a polyhedron is called regular if its automorphism group acts transitively on its flags (incidence triples of vertex, edge and face).

Regular maps and their groups play a central role in classical complex analysis and algebraic geometry (e.g. Riemann surfaces, automorphic functions, Poincaré model). Hence regular polyhedra can be interpreted as 3D geometric models or visualizations of regular maps, and they are closely related to the Platonic solids.

The geometric (or Euclidean) symmetry group of the polyhedron is the group of isometries of $\mathbb{E}^{3}$ stabilizing the polyhedron. It is a subgroup of the automorphism group; to be precise, the automorphism group has a (proper, or improper) subgroup that is isomorphic to the geometric symmetry group. For a combinatorially regular polyhedron the geometric symmetry group is, in general, much smaller than the automorphism group; they coincide only in the case of Platonic solids. For any polyhedron with given combinatorial structure we tacitly assume that it has maximal geometric symmetry.

Polyhedra which have the geometric rotation or full symmetry group of a Platonic solid deserve particular interest. They are called Leonardo polyhedra, because Leonardo was the first to draw such polyhedra in Luca Pacioli's book [19] in 1500-1503 (see also [6] and [12]). It is easy to check that the polyhedra in this book are neither equivelar nor regular. Leonardo also drew some polyhedra with lower symmetry groups (e.g. dihedral), but we only use the name for Platonic symmetries.

Obviously there are no Leonardo polyhedra of genus $g=1$, because tori can have at most dihedral symmetry. For similar reasons there are no Leonardo polyhedra with $g=2$. For $g=3$ there are some with tetrahedral symmetry.

\section{Regular and equivelar Leonardo polyhedra}

Regular Leonardo polyhedra seem to be very rare. Only six are known yet. The first four are Coxeter's regular skew polyhedra [5], first discovered by Coxeter in 1937 and partially by Alicia Boole Stott already in 1913 [1]. There is one dual pair of genus $g=6$, with tetrahedral symmetry and of type $\{4,6\}$ and $\{6,4\}$, and one dual pair of genus $g=73$, with octahedral symmetry and of type $\{4,8\}$ and $\{8,4\}$. (In standard notation: $\{4,6 \mid 3\}$, $\{6,4 \mid 3\},\{4,8 \mid 3\}$ and $\{8,4 \mid 3\}$, cf. $[5,15,21]$.) The spines of Coxeter's regular skew polyhedra are isomorphic to the 1-skeletons of the regular 4-simplex or the regular 24-cell, i.e. the only self-dual regular 4-polytopes. We note that the term spine, borrowed from topology, is meant here as a graph, embedded in $\mathbb{E}^{3}$, such that its regular neighbourhood [18] is a 3-manifold with boundary, and the boundary of this manifold is just our polyhedron.

Furthermore, there is the polyhedral realization [20] of Felix Klein's regular map of genus 3 with tetrahedral rotation group and of type $\{3,7\}$. Its dual with non-convex heptagons was recently discovered [13], but here we only consider polyhedra with convex faces.

The sixth regular polyhedron is the realization of the regular map of Fricke and Klein from 1890. This polyhedron was found by Grünbaum and Shephard in 1984 [11], because of its vertex-transitivity. But its regularity was only recently discovered [2]. It is of type 
$\{3,8\}$, with genus 5 , and it has octahedral rotation symmetry.

The spines of these last two polyhedra are isomorphic to the 1-skeleton of the tetrahedron or the cube, hence of convex 3-polytopes in both cases. No other regular Leonardo polyhedra are known yet.

In this paper we construct series of equivelar polyhedra, which are related to the previous 6 regular polyhedra:

Theorem 2.1. There are infinite series of equivelar Leonardo polyhedra with tetrahedral, octahedral and dodecahedral symmetry group and of Schläfli type $\{3,7\},\{3,8\},\{3,9\}$, $\{4,6\}$ and $\{6,4\}$, and whose spine is isomorphic to the 1-skeleton of a convex 3 -or 4 polytope.

Remark 2.2. The result shows that there are infinite series of equivelar polyhedra, which are closely related to the regular Leonardo polyhedra. Only the types $\{4,8\}$ and $\{8,4\}$ are missing.

Remark 2.3. In the previous papers [9] and [23] the authors provided infinite series of equivelar Leonardo polyhedra of type $\{4,6\}$ and $\{6,4\}$. But these were of very different spatial structure than the six known regular ones, as they are built up of connected shells (like an onion). The search for closer equivelar analogues was one motivation for this paper. The other one was the recently discovered regularity [2] of the Grünbaum-Shephard polyhedron.

Among the polyhedra of "small" Schläfli-type (i.e. those with $p+q<12$ ) the equivelar polyhedra of type $\{4,5\}$ and $\{5,4\}$ differ from the others, as follows. The only known regular polyhedra of these types have genus 5 and a small symmetry group of order 4 , namely $\left(\mathbb{Z}_{2} \times \mathbb{Z}_{2}\right)$ (cf. [17] and [14]).

The equivelar Leonardo polyhedra of this type also differ from the others as the following result shows.

Theorem 2.4. a) Type $\{4,5\}$ : There exist four infinite series of equivelar Leonardo polyhedra with the following genera and symmetry groups:

- $g=1+6 k$ with tetrahedral symmetry group, in two non-isomorphic versions;

- $g=1+12 k$ with octahedral symmetry group;

- $g=1+30 k$ with icosahedral symmetry group $(k=1,2, \ldots)$.

b) Type $\{5,4\}$ : There exist equivelar Leonardo polyhedra with the following genera and symmetry groups:

- $g=13,31$ with tetrahedral symmetry group;

- $g=7,13,25,97,289$ with octahedral symmetry group;

- $g=31,61,3601$ with icosahedral symmetry group.

In [22] there is a pair of equivelar Leonardo polyhedra of type $\{4,5\}$ and $\{5,4\}$ with $g=7$ and octahedral symmetry, and in Figure 1 we show a new example of type $\{4,5\}$ with $g=19$ and icosahedral (rotation) symmetry. This polyhedron consists of an outer and an inner shell, homothetic to each other and positioned concentrically. Both of them are composed of 60 quadrangular faces; besides, there are 20 triangular holes in each. The two 


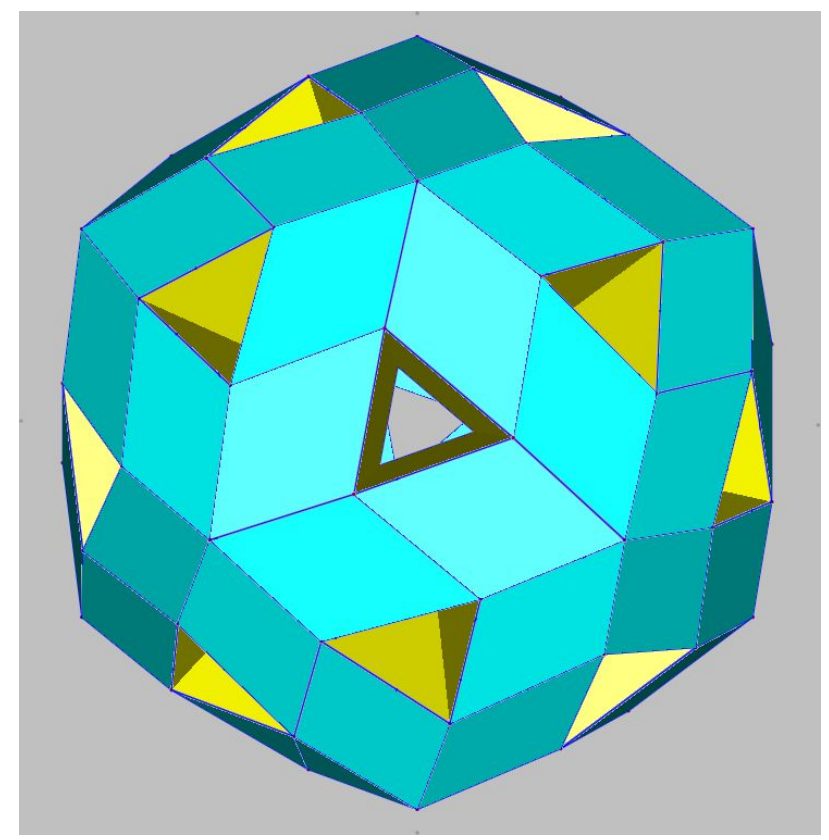

Figure 1: The Leonardo polyhedron of type $\{4,5 ; 19\} ; f=36(4,10,5)$.

shells are joined by triangular prismatic tubes along these holes. Since this polyhedron has shortest non-0-homotopic paths of length 3 and 4, it is not regular. It follows from Conder's list of regular maps [4] that for $g=19$ there is precisely one regular map of type $\{4,5\}$. So the only possible Leonardo polyhedron of such type would be a realization of this map, and it is an open question if it exists or not.

We note also that the polyhedron of type $\{5,4 ; 7\}$ with octahedral symmetry group was found already in 1983 (see [22], Figure 2). In [9] it is constructed in a slightly different way. The other related types are new and here we show in Figure 2 the example with genus 13.

\section{Proof for the existence of equivelar series}

In this section we prove Theorems 2.1 and 2.4 by constructing the polyhedra in question.

\section{Proof of Theorem 2.1}

CONSTRUCTION for Schläfli type $\{3,7\}$.

The construction was already done in [16], but without any symmetry assumptions. We split our proof into three parts.

First we show that there are infinitely many simple convex 3-polytopes, i.e. such that all their vertices are 3-valent, for each of the required symmetry groups. The first polytopes of this type are the tetrahedron, the cube and the dodecahedron. They have 4, 8 and 20 3 -valent vertices, respectively. We now continue by induction. 


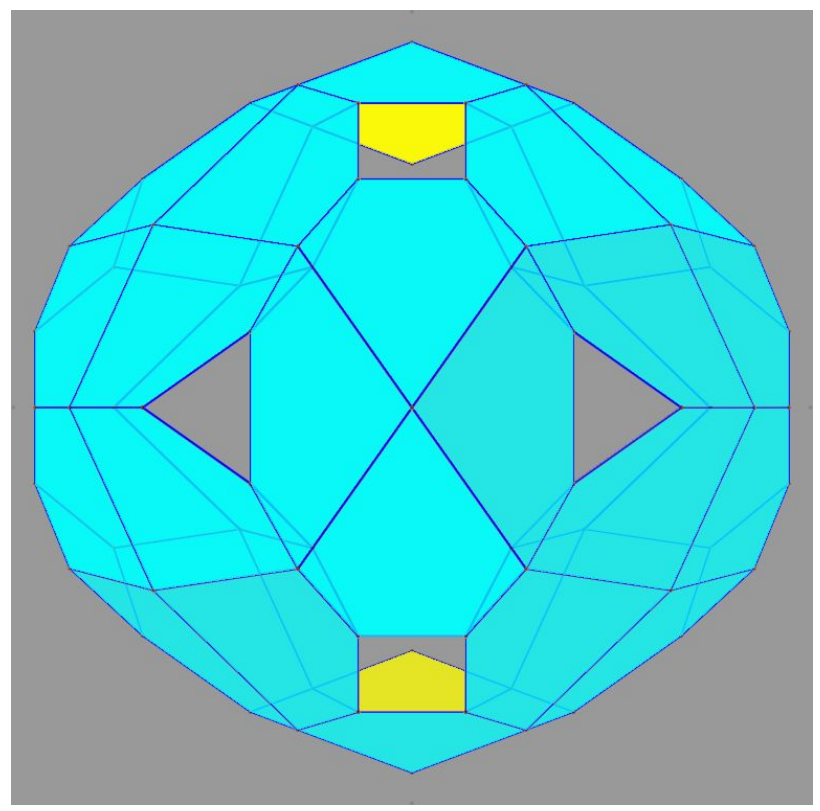

Figure 2: The Leonardo polyhedron of type $\{5,4 ; 13\} ; f=24(5,10,4)$.

For a simple convex polytope with $v$ vertices we cut off these vertices by a plane each, such that no cuts intersect and that the global symmetry is preserved. We obtain a new convex 3-polytope with the same symmetry group and with $3 v$ vertices, all of them 3valent. Clearly this polytope is not equivelar, because it contains faces of different type.

The second step is to construct from each of these polytopes a new one with 5-valent vertices.

For each of the convex polytopes with $3 v$ vertices we make the following operation. We shrink all faces by the same factor, such that each face remains in its given affine hull. Hence they are disjoint. Now we take the convex hull of the system of these new polygons, so that we obtain a new convex polytope with 4 -valent vertices. Each vertex of the former polytope corresponds to a triangle, so does each edge to a quadrangle, and the vertex-number of the new polytope is $9 v$.

We now split the new quadrangles by a diagonal into two triangles in the right order, such that the global symmetry is preserved. More precisely, by this operation the full symmetry group is lost and reduced to the rotation subgroup of index two. We now have a convex 3-polytope with 5-valent vertices and the required symmetry.

The third step leads us to the construction of tunnels and the required polyhedron of type $\{3,7\}$. First we take the boundary complex of our polytope, and put a smaller copy of this complex into the former one with the same centre and orientation. Then we delete in both objects the shrinked polygons. The remaining faces are all triangles. We now connect any two corresponding holes by tunnels, built up of quadrangles. Again we split each of these quadrangles by a diagonal in the right order, so that the symmetry is preserved. We obtain a polyhedron of Schläfli type $\{3,7\}$ with the required symmetry. Finally, by a slight 
rotation of the holes (originating from the shrinked faces), each within its affine hull and to different extent in the two spherical complexes, it is ensured that no adjacent triangles are coplanar (this can also be done in symmetry-preserving way).

\section{CONSTRUCTION for Schläfli types $\{4,6\}$ and $\{6,4\}$.}

The construction was already described in [16], although without any symmetry considerations. In order to make the paper self-contained, we sketch the proof for the $\{6,4\}$ series. The dual $\{4,6\}$ series are constructed similarly. Let $P$ be one of the simple 3polytopes with Platonic symmetry group obtained in the first step of our former proof and let $S D$ be the Schlegel diagram of the 4-prism with base $P$ such that it is in one of the Platonic bases of the prism. All vertices of $S D$ are 4-valent. We take the midpoints of all edges of $S D$, and then the convex hull of the midpoints of any four edges which are incident to a vertex of $S D$. Thus to each vertex of $S D$ corresponds a 3-simplex and each vertex of a simplex is shared with a vertex of a neighboring simplex. Now we enlarge each simplex by the same factor $1+\varepsilon, \varepsilon>0$ sufficiently small. We delete those parts of the simplices which lie inside another simplex and obtain a polyhedron of type $\{6,4\}$ with the required symmetry properties.

\section{CONSTRUCTION for Schläfli-types $\{3,8\}$ and $\{3,9\}$.}

From each polyhedron of type $\{6,4\}$ we obtain one of type $\{3,8\}$ as follows. In each hexagon one connects a triplet of non-consecutive vertices by segments and obtains a tiling of the hexagon into 4 triangles. If one does this in the right order on the whole $\{6,4\}$ polyhedron, one obtains the required $\{3,8\}$ polyhedron.

The $\{3,9\}$ series is obtained from the $\{4,6\}$ series as follows. Each quadrangle can be divided into two triangles by a diagonal. If one does this in the right order on the whole polyhedron, one obtains the required $\{3,9\}$ polyhedron. The crucial point for this procedure (which was already described in [16]) is the fact that, when applied to any polyhedron of type $\{p, q\}$, the valency $q$ of the vertices is even.

\section{Proof of Theorem 2.4}

\section{CONSTRUCTION for Schläfli-type $\{4,5\}$.}

Start from two distinct types of Archimedean polyhedra, $P_{1}$ and $P_{2} . P_{1}$ is the truncated octahedron with six square faces and eight hexagonal faces. $P_{2}$ is the rhombicuboctahedron, which has $6+12$ square faces and eight triangular faces. For the following construction it is crucial that $P_{1}$ has only 3 -valent vertices, and $P_{2}$ only 4 -valent vertices. Note that both the octuple of the hexagonal faces of $P_{1}$ and the octuple of the triangular faces of $P_{2}$ decomposes to two disjoint classes. In each case such a class is a quadruple forming an orbit under the action of the tetrahedral symmetry group (a subgroup of the octahedral symmetry group of these polyhedra). Delete these hexagonal and triangular faces, and denote the complexes obtained in this way by $P_{1}^{\prime}$ and $P_{2}^{\prime}$, respectively. Take $2 k(k \geq 2)$ concentric and homothetic copies of $P_{1}^{\prime}$. We call them shells of our polyhedron under construction. Now join the holes of the neighbouring shells by hexagonal prismatic tubes. The tubes are arranged so that each intermediate shell is joined to its outer or inner neighbour, in both cases using four tubes and using holes that belong to the same class (but different in the two cases). The innermost shell is joined to the outermost shell. To avoid undesirable contacts, 
the holes of the outermost shell are shrinked to a suitable size with respect to those of the intermediate shells, while keeping the symmetry. For $P_{2}^{\prime}$, the construction is the same with the only difference that here one uses triangular tubes. We obtain two different infinite series of polyhedra, both of the desired Schläfli type, and with the (full) tetrahedral symmetry group and genus $g=1+6 k(k=1,2, \ldots)$.

Consider again the four orbits of faces of the rhombicuboctahedron with respect to the action of the tetrahedral group. Clearly, analogous polyhedra can be constructed, likewise with four orbits of faces with respect to the octahedral and the icosahedral group. In the octahedral case these orbits are 6 squares, 8 regular triangles, 12 rhombi and 24 rectangles, while in the icosahedral case there are 12 regular pentagons, 20 regular triangles, 30 squares and 60 symmetric trapezia. In both cases all the vertices are 4-valent (and the polar dual is such that all the faces are kite-shaped and form two orbits). Now deleting the square and triangular faces in the the octahedral case, and the non-quadrangular faces in the icosahedral case, and applying an analogous construction as above, one obtains the desired infinite series of polyhedra with genus $g=1+12 k$ and $g=1+30 k$, respectively. The starting member of the icosahedral series is shown in Figure 3.

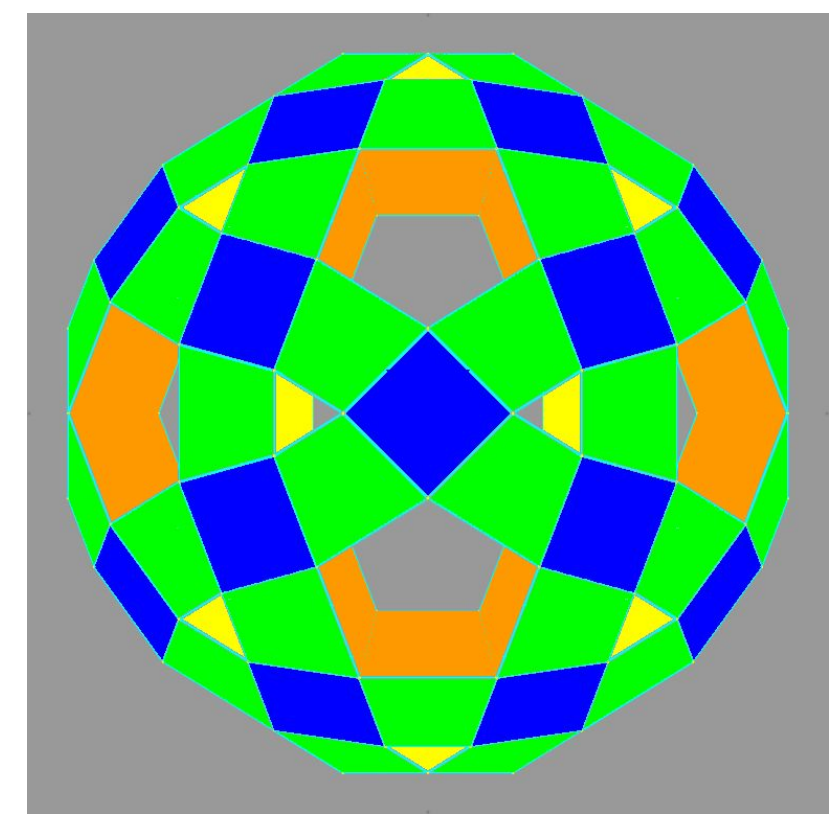

Figure 3: The Leonardo polyhedron of type $\{4,5 ; 31\} ; f=60(4,10,5)$.

Construction for Schläfli-type $\{5,4\}$.

Let $P$ be a polyhedron satisfying the following conditions:

(1) the symmetry group $G(P)$ of $P$ is equal to the full symmetry group of one of the Platonic solids; 
(2) $G(P)$ is transitive on the faces of $P$;

(3) the faces of $P$ are quadrangles;

(4) each edge of $P$ is contained in one of the mirror planes determined by $G(P)$.

It is easy to see that $P$ is combinatorially equivalent to one of the following five polyhedra (cf. [8]):

- cube;

- rhombic dodecahedron;

- rhombic triacontahedron;

- deltoidal icositetrahedron (dual of the rhombicuboctahedron) (see e.g. Figure 3 in [9];

- deltoidal hexecontahedron (dual of the Archimedean polyhedron called rhombicosidodecahedron) (see e.g. Figure 5 in [9] or Figure 9 in [7]).

Put on each of the quadrangular faces of $P$ a bipyramid, each pairwise congruent, such that the midpoints of the edges of the face form the basal vertices of the bipyramid. Then enlarge each bipyramid from the centre of its own base by the same factor $1+\epsilon, \epsilon>0$ sufficiently small. Delete now those parts of the bipyramids which lie inside another bipyramid (along with the original faces of $P$ ). One obtains a polyhedron of the desired Schläfli type such that its symmetry group remains the same as that of the example of $P$ we started from. The genus of this polyhedron is $g=f_{2}(P)+1$, where $f_{2}(P)$ is the number of the faces of $P$; hence the genera in the five cases above are 7,13,31, 25 and 61, respectively. Finally, we note that there is a polyhedron called deltoidal dodecahedron, combinatorially equivalent to the rhombic dodecahedron but with tetrahedral symmetry (a well-known figure in geometric crystallography, see e.g. [10]). It also satisfies the conditions above. Hence starting from it, our construction provides the tetrahedrally symmetric polyhedron with $g=13$.

The conditions (1-4) above can be suitably modified such that they are satisfied by equivelar polyhedra with quadrangular faces and with spine isomorphic to the 1-skeleton of a regular 4-polytope $Q$. (Two of these polyhedra are even regular, namely that of type $\{4,6 ; 6\}$ and $\{4,8 ; 73\}$ [21].) Thus, performing the construction in $\mathbb{E}^{4}$, then taking a suitable projection to $\mathbb{E}^{3}$, one obtains Leonardo polyhedra of the following genera: $g=31$ ( $Q$ is the regular 4-simplex), $g=97$ ( $Q$ is either the 4-cube or the regular 16-cell), $g=289$ ( $Q$ is the regular 24-cell) and $g=3601$ ( $Q$ is either the regular 120-cell or the 600-cell).

In conclusion, we present a sporadic example of a polyhedron of type $\{3,8 ; 7\}$, which differs in its structure from those in Theorem 2.1. It is constructed from two solids $P$ and $P^{\prime}$, such that $P^{\prime}$ is a non-convex version of the convex 3-polytope $P$; their boundary are combinatorially equivalent to each other, and have the $f$-vector $f=(30,84,56)$. The size and shape of the bounding polyhedra is adjusted so that deleting a whole 8-element orbit of faces from both, the complexes obtained in this way can be glued together along the holes, thus forming the outer and inner shell of a new polyhedron. This polyhedron has octahedral rotation symmetry; it is shown in Figure 4a, and its inner shell in Figure 4b. 


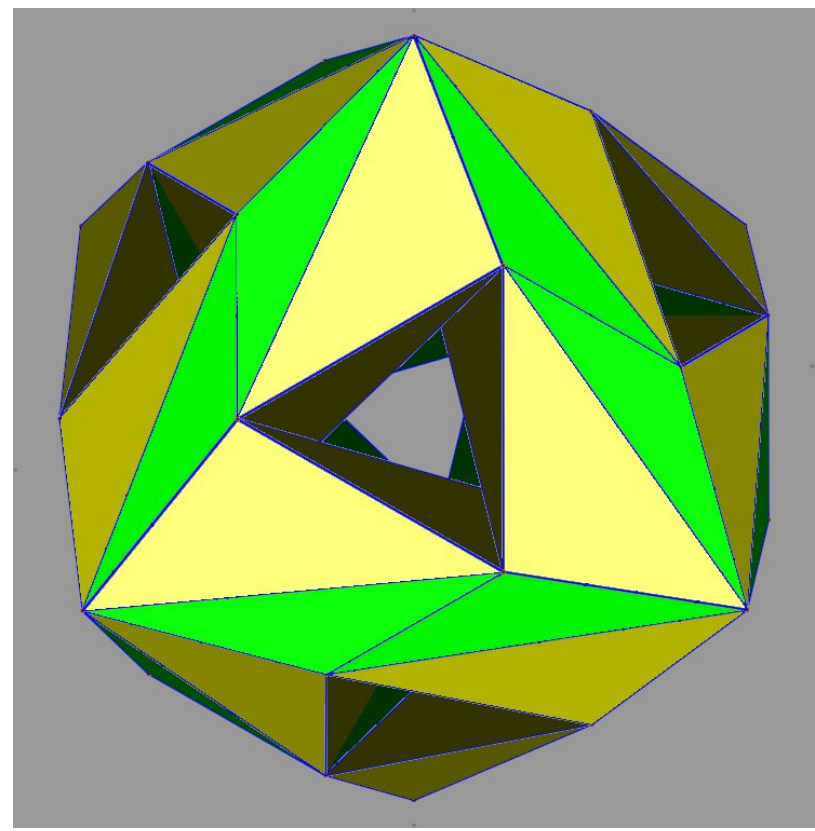

(a) The whole polyhedron.

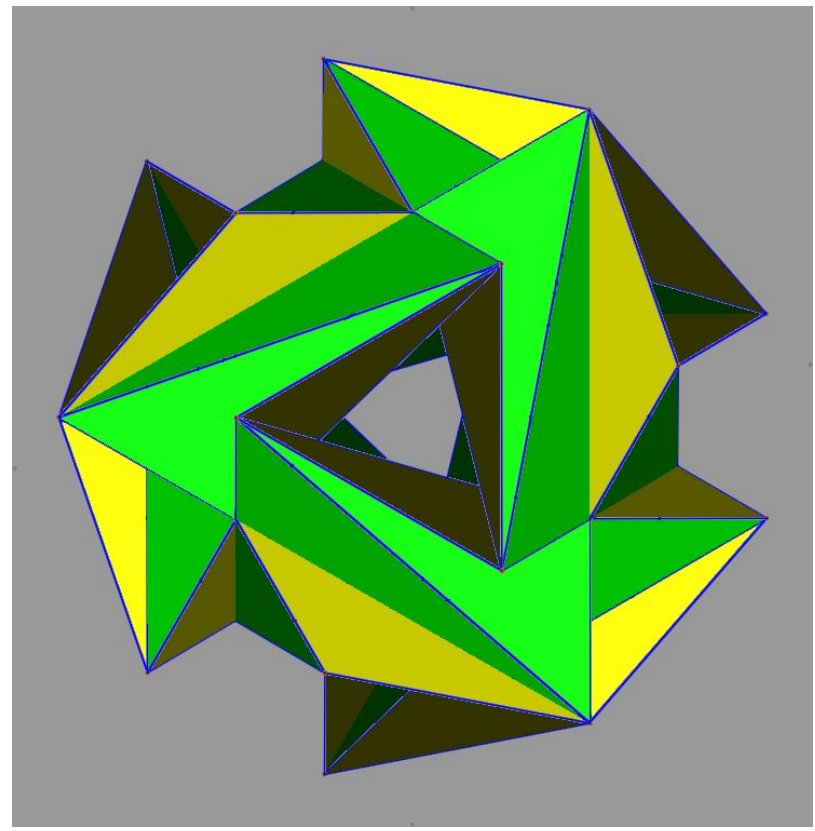

(b) The inner shell of the polyhedron.

Figure 4: The Leonardo polyhedron of type $\{3,8 ; 7\} ; f=12(3,12,8)$. 


\section{References}

[1] A. Boole Stott, Geometrical deduction of semiregular from regular polytopes and space fillings, Verhandelingen der Koninklijke Akademie van Wetenschappen te Amsterdam 11 (1913), 3-24.

[2] U. Brehm, B. Grünbaum and J. M. Wills, Polyhedral realization of the regular Fricke-Klein map of genus 5 , in preparation.

[3] U. Brehm and J. M. Wills, Polyhedral manifolds, in: P. M. Gruber and J. M. Wills (eds.), Handbook of Convex Geometry, North-Holland, Amsterdam, 1993, 535-554.

[4] http://www.math.auckland.ac.nz/ conder/

[5] H. S. M. Coxeter, Regular skew polyhedra in three and four dimensions and their topological analogues, Proc. London Math. Soc. 43 (1937), 33-62. Reprinted in: H. S. M. Coxeter, Twelve Geometric Essays, Southern Illinois University Press, Carbondale, 1968, 75-105.

[6] J. V. Field, Rediscovering the Archimedean polyhedra: Piero della Francesca, Luca Pacioli, Leonardo da Vinci, Albrecht Dürer, Daniele Barbaro, and Johannes Kepler, Arch. Hist. Exact Sci. 50 (1997), 241-289.

[7] G. Gévay, Icosahedral morphology, in: I. Hargittai (ed.), Fivefold Symmetry, World Scientific, Singapore, 1992, 177-203.

[8] G. Gévay, Kepler hypersolids, in: K. Böröczky and G. Fejes Tóth (eds.), Intuitive Geometry, North-Holland, Amsterdam - Math. Soc. János Bolyai, Budapest, 1994, 119-129.

[9] G. Gévay, Constructions for some classes of equivelar skew polyhedra, Symmetry Cult. Sci. 22, No. 3-4 (2011), 327-345.

[10] G. Gévay and K. Miyazaki, Some examples of semi-nodal perfect 4-polytopes, Publ. Math. Debrecen 63-4 (2003), 715-735.

[11] B. Grünbaum and G. C. Shephard, Polyhedra with transitivity properties, C. R. Math. Rep. Acad. Sci. Canada 6 (1984), 61-66.

[12] D. Huylebrouk, Lost in triangulation: Leonardo da Vinci's mathematical slip-up, Scientific American, March 29, 2011.

[13] D. I. McCooey, A non-self-intersecting polyhedral realization of the all-heptagon Klein map, Symmetry Cult. Sci. 20 (2009), 247-268.

[14] P. McMullen, E. Schulte and J. M. Wills, Infinite series of combinatorially regular polyhedra in three-space, Geom. Dedicata 26 (1988), 299-307.

[15] P. McMullen and E. Schulte, Abstract Regular Polytopes, Cambridge University Press, Cambridge, 2002.

[16] P. McMullen, C. Schulz and J. M. Wills, Equivelar polyhedral manifolds in $\mathbb{E}^{3}$, Israel J. Math. 41 (1982), 331-346.

[17] P. McMullen, C. Schulz and J. M. Wills, Polyhedral 2-manifolds in $\mathbb{E}^{3}$ with unusually large genus, Israel J. Math. 46 (1983), 127-144.

[18] E. E. Moise, Geometric Topology in Dimensions 2 and 3, Springer-Verlag, New York, 1977.

[19] L. Pacioli, De Divina Proportione (Disegni di Leonardo da Vinci 1500-1503), Faksimile Dominiani, Como, 1967.

[20] E. Schulte and J. M. Wills, A polyhedral realization of Felix Klein's map $\{3,7\}_{8}$ on a Riemann surface of genus 3, J. London Math. Soc. 32 (1985), 539-547.

[21] E. Schulte and J. M. Wills, On Coxeter's regular skew polyhedra, Discrete Math. 60 (1986), 253-262. 
[22] J. M. Wills, Semi-Platonic manifolds, in: P. M. Gruber and Jörg M. Wills (eds.), Convexity and its Applications, Birkhäuser, Basel, 1983, 413-421.

[23] J. M. Wills, Combinatorially regular Leonardo polyhedra, Symmetry Cult. Sci. 22, No. 1-2 (2011), 55-64. 